I. ISHIHARA

KODAI MATH. J.

2 (1979), $171-186$

\title{
ANTI-INVARIANT SUBMANIFOLDS OF A SASAKIAN SPACE FORM
}

\author{
BY IKUO ISHIHARA
}

\section{$\S 1$. Introduction.}

In a previous paper [8] we studied anti-invariant submanifolds in a Kähler manifold, especially in a complex space form. In the present paper we shall study anti-invariant submanifolds of a Sasakian manifold, especially those of a Sasakian space form, in the same way as taken in [8].

An $(n+1)$-dimensional Riemannian manifold $M$ isometrically immersed in a $(2 m+1)$-dimensional Sasakian manifold $\bar{M}$ with structure tensors $(\phi, \xi, \eta, \bar{g})$ is said to be anti-invariant (resp. invariant) if $\phi T_{p}(M) \subset T_{p}(M)^{\perp}\left(\operatorname{resp} . \phi T_{p}(M) \subset\right.$ $T_{p}(M)$ ) for each point $p$ of $M$, where $T_{p}(M)$ and $T_{p}(M)^{\perp}$ denote respectively the tangent and the normal spaces to $M$ at $p$. Thus in an anti-invariant submanifold $\phi X$ is normal to $M$ for any vector $X$ tangent to $M$. Since $\phi$ is necessarily of rank $2 m$, we have $n \leqq(2 m+1)-(n+1)$ which implies $n \leqq m$. In the present paper, we assume that for any anti-invriant submanifold $M$ we consider the structure vector field $\xi$ of the ambient manifold is tangent to $M$ everywhere.

When for an anti-invariant submanifold $M$ the structure vector field $\xi$ of the ambient manifold $\bar{M}$ is tangent to $M$, then each of the following assumptions (a), (b), (c) is not meaningful: (a) the second fundamental form is parallel ; (b) the mean curvature vector is parallel; (c) the connection induced in the normal bundle is flat. So, in the present paper, we shall replace the assumptions (a), (b), (c) respectively by new but rather weaker assumptions $\left(a^{\prime}\right),\left(b^{\prime}\right)$, $\left(c^{\prime}\right)$ as follows: $\left(a^{\prime}\right)$ the second fundamental form is pseudo-parallel ; $\left(b^{\prime}\right)$ the mean curvature vector is pseudo-parallel; $\left(c^{\prime}\right)$ the connection induced in the normal bundle is pseudo-flat (see Lemmas 3.2, 3.3 and 4.1).

\section{§. Sasakian manifolds.}

First, we would like to recall definitions and some fundamental properties of Sasakian manifolds. Let $\bar{M}$ be a $(2 m+1)$-dimensional differentiable manifold of class $C^{\infty}$ and $\phi, \xi, \eta$ be a tensor field of type $(1,1)$, a vector field, a 1 -form on $\bar{M}$ respectively such that

Received December 17, 1977 


$$
\phi^{2}=-I+\eta \otimes \xi, \quad \phi \xi=0, \quad \eta(\phi X)=0, \quad \eta(\xi)=1
$$

for any vector field $X$ on $\bar{M}$, where $I$ denotes the identity tensor of type $(1,1)$. Then $\bar{M}$ is said to admit an almost contact structure $(\phi, \xi, \eta)$ and called an almost contact manifold. The almost contact structure is said to be normal if

$$
N+d \eta \otimes \xi=0,
$$

where $N$ denotes the Nijenhuis tensor formed with $\phi$. If there is given in $\bar{M}$ a Riemannian metric $\bar{g}$ satisfying

$$
\bar{g}(\phi X, \phi Y)=\bar{g}(X, Y)-\eta(X) \eta(Y), \quad \eta(X)=\bar{g}(X, \xi)
$$

for any vector fields $X$ and $Y$ on $\bar{M}$, then the set $(\phi, \xi, \eta, \bar{g})$ is called a almost contact metruc structure and $\bar{M}$ an almost contact metruc manifold. If

$$
d \eta(X, Y)=\bar{g}(\phi X, Y)
$$

for any vector fields $X$ and $Y$ an $\bar{M}$, then the almost contact metric structure is called a contact metric structure. If the structure is moreover normal, then the contact metric structure is called a Sasakian structure and $\bar{M}$ a Sasakian manufold. As is well known, in a Sasakian manifold $\bar{M}$ with structure $(\phi, \xi, \eta, \bar{g})$

$$
\bar{\nabla}_{X} \xi=\phi X, \quad\left(\bar{\nabla}_{X} \phi\right) Y=-\bar{g}(X, Y) \xi+\eta(Y) X
$$

are established for any vector fields $X$ and $Y$ on $\bar{M}$, where $\bar{\nabla}$ denotes the operator of covariant differentiantion with respect to $\bar{g}$.

A plane section $\sigma$ in the tangent space $T_{p}(\bar{M})$ of a Sasakian manifold $\bar{M}$ at $p$ is called a $\phi$-section if it is spanned by vectors $X$ and $\phi X$, where $X$ is assumed to be orthogonal to $\xi$. The sectional curvature $K(\sigma)$ with respect to a $\phi$-section $\sigma$ is called a $\phi$-sectional curvature. When the $\phi$-sectional curvature $K(\sigma)$ is independent of the $\phi$-section $\sigma$ at each point of $\bar{M}$, as is well known, the function $K(\sigma)$ defined in $\bar{M}$ is necessarily a constant $c$. A Sasakian manifold $\bar{M}$ is called a Sasakian space form and denoted by $\bar{M}(c)$ if it has constant $\phi$ sectional curvature $c$ (see [4]). The curvature tensor $K$ of a Sasakian space form $\bar{M}(c)$ is given by

$$
\begin{aligned}
K(X, Y) Z= & \frac{1}{4}(c+3)(\bar{g}(Y, Z) X-\bar{g}(X, Z) Y)-\frac{1}{4}(c-1)(\eta(Y) \eta(Z) X \\
& -\eta(X) \eta(Z) Y+\bar{g}(Y, Z) \eta(X) \xi-\bar{g}(X, Z) \eta(Y) \xi \\
& -\bar{g}(\phi Y, Z) \phi X+\bar{g}(\phi X, Z) \phi Y+2 \bar{g}(\phi X, Y) \phi Z) .
\end{aligned}
$$

ExAmple 1. Let $S^{2 n+1}$ be a $(2 n+1)$-dimensional unit sphere, i. e.,

$$
S^{2 n+1}=\left\{z \in C^{n+1}:|z|=1\right\},
$$

where $C^{n+1}$ is a complex $(n+1)$-space. For any point $z \in S^{2 n+1}$, we put $\xi=J z, J$ being the complex structure of $C^{n+1}$. Considering the orthogonal projection 


$$
\pi: T_{z}\left(C^{n+1}\right) \longrightarrow T_{z}\left(S^{2 n+1}\right),
$$

at each point $z$ in $S^{2 n+1}$ and putting $\phi=\pi \circ J$, we have a Sasakian structure $(\phi, \xi, \eta, g)$ on $S^{2 n+1}$, where $\eta$ is a 1 -form dual to $\xi$ and $g$ the standard metric tensor field on $S^{2 n+1}$. Obviously, $S^{2 n+1}$ is of constant $\phi$-sectional curvature 1 .

EXAMPLE 2. Let $E^{2 n+1}$ be a Euclidean space with cartesian coordinates $\left(x^{1}, \cdots, x^{n}, y^{1}, \cdots, y^{n}, z\right)$. Then a Sasakian structure on $E^{2 n+1}$ is defined by $\phi, \xi, \eta$ and $g$ such that

$$
\begin{gathered}
\xi=(0, \cdots, 0,2), \quad 2 \eta=\left(-y^{1}, \cdots,-y^{n}, 0, \cdots, 0,1\right), \\
\left(g_{A B}\right)=\left(\begin{array}{ccc}
\frac{1}{4}\left(\delta_{\imath \jmath}+y^{\imath} y^{\jmath}\right) & 0 & -\frac{1}{4} y^{2} \\
0 & \frac{1}{4} \delta_{\iota \jmath} & 0 \\
-\frac{1}{4} y^{\imath} & 0 & \frac{1}{4}
\end{array}\right), \\
\left(\phi_{B}^{A}\right)=\left(\begin{array}{ccc}
0 & \delta_{\jmath}^{\prime} & 0 \\
-\delta_{\jmath}^{\imath} & 0 & 0 \\
0 & y^{\jmath} & 0
\end{array}\right) .
\end{gathered}
$$

Then $E^{2 n+1}$ with such a structure $(\phi, \xi, \eta, g)$ is of constant $\phi$-sectional curvature -3 and denoted by $E^{2 n+1}(-3)$.

\section{$\S 3$. Fundamental properties of anti-invariant submanifolds.}

Let $\bar{M}$ be a Sasakian manifold of dimension $2 m+1$ with structure tensors $(\phi,, \xi, \eta, \bar{g})$. An $(n+1)$-dimensional Riemannian manifold $M$ isometrically immersed in $\bar{M}$ is said to be anti-invariant in $\bar{M}$ if $\phi T_{p}(M) \subset T_{p}(M)^{\perp}$ for each point $p$ of $M$. Throughout the paper, we now restrict ourselves only to antiinvariant submanifolds of a Sasakian manifold such that the structure vector field $\xi$ of the ambient manifold is tangent to the submanifolds.

We choose a local field of orthonormal frames $e_{0}=\xi, e_{1}, \cdots, e_{n} ; e_{n+1}, \cdots, e_{m}$; $e_{1^{*}}=\phi e_{1}, \cdots, e_{n^{*}}=\phi e_{n} ; e_{(n+1)^{*}}=\phi e_{n+1}, \cdots, e_{m^{*}}=\phi e_{m}$ in $\bar{M}$ in such a way that $e_{0}, e_{1}, \cdots, e_{n}$ are along $M$ tangent to $M$. Taking such a field of frames of $\bar{M}$, we denote the dual coframes by $\omega^{0}=\eta, \omega^{1}, \cdots, \omega^{n} ; \omega^{n+1}, \cdots, \omega^{m} ; \omega^{1^{*}}, \cdots, \omega^{n^{*}}$; $\omega^{(n+1)^{*}}, \cdots, \omega^{m^{*}}$. Unless otherwise stated, let the ranges of indices be as follows:

$A, B, C, D=0,1, \cdots, m, 1^{*}, \cdots m^{*}$,

$\imath, j, k, l, s, t=0,1, \cdots, n$,

$x, y, z, v, w=1, \cdots, n$,

$a, b, c, d=n+1, \cdots, m, 1^{*}, \cdots m^{*}$,

$\alpha, \beta, \gamma=n+1, \cdots, m$,

$\lambda, \mu, \nu=n+1, \cdots, m,(n+1)^{*}, \cdots, m^{*}$, 
and use the so-called summation convention for these systems of indices. Then the structure equations of the Riemannian manifold $\bar{M}$ are given by

$$
\begin{gathered}
d \omega^{A}=-\omega_{B}^{A} \wedge \omega^{B}, \quad \omega_{B}^{4}+\omega_{A}^{B}=0, \\
d \omega_{B}^{A}=-\omega_{C}^{A} \wedge \omega_{B}^{C}+\Phi_{B}^{A}, \quad \Phi_{B}^{A}=\frac{1}{2} K_{B C D}^{A} \omega^{C} \wedge \omega^{D},
\end{gathered}
$$

where $K_{B C D}^{A}$ are components of the curvature tensor of $\bar{M}$ with respect to $\left\{e_{A}\right\}$ and $\omega_{B}^{4}$ satisfy

$$
\begin{array}{llll}
\omega_{y}^{r}=\omega_{y^{*}}^{r^{*}}, & \omega_{y}^{r^{*}}=\omega_{x}^{y^{*}}, & \omega^{x}=\omega_{0}^{r^{*}}, & \omega^{x^{*}}=-\omega_{0}^{r}, \\
\omega_{\beta}^{r}=\omega_{\beta^{*}}^{\gamma^{*}}, & \omega_{\beta}^{r^{*}}=\omega_{\alpha}^{\beta^{*}}, & \omega^{\alpha}=\omega_{0}^{\alpha^{*}}, & \omega^{\alpha^{*}}=-\omega_{0}^{\alpha}, \\
\omega_{\alpha}^{r}=\omega_{\alpha^{*}}^{r^{*}}, & \omega_{\alpha}^{r^{*}}=\omega_{x}^{\alpha^{*}} . &
\end{array}
$$

Thus we have along $M$

$$
\omega^{a}=0,
$$

which implies $0=d \omega^{a}=-\omega_{i}^{\tau} \wedge \omega^{2}$ alng $M$. Thus, by Cartan's lemma, we obtain along $M$

$$
\omega_{\imath}^{a}=h_{\imath j}^{a} \omega^{j}, \quad h_{\imath j}^{a}=h_{j i}^{a},
$$

which imply the following structure equations of the submanifold $M$ :

$$
\begin{aligned}
& d \omega^{2}=-\omega_{j}^{2} \wedge \omega^{j}, \quad \omega_{j}^{2}+\omega_{i}^{j}=0, \\
& d \omega_{\jmath}^{\imath}=-\omega_{k}^{i} \wedge \omega_{\jmath}^{k}+\Omega_{\jmath}^{i}, \quad \Omega_{j}^{\imath}=\frac{1}{2} R_{j k l}^{\prime} \omega^{k} \wedge \omega^{l}, \\
& R_{j k l}^{\imath}=K_{j k l}^{i}+\sum_{a}\left(h_{i k}^{a} h_{j l}^{a}-h_{i l}^{a} h_{j k}^{a}\right), \\
& d \omega_{b}^{a}=-\omega_{c}^{a} \wedge \omega_{b}^{r}+\Omega_{b}^{a}, \quad \Omega_{b}^{n}=\frac{1}{2} R_{b k l}^{a} \omega^{k} \wedge \omega^{l}, \\
& R_{b k l}^{a}=K_{b k l}^{a}+\sum_{\imath}\left(h_{i k}^{a} h_{i l}^{b}-h_{i l}^{a} h_{i k}^{b}\right),
\end{aligned}
$$

where $R_{j k l}^{\prime}$ are components of the curvature tensor of $M$ with respect to $\left\{e_{i}\right\}$ and $R_{b k l}^{a}$ components of the curvature tensor of the normal bundle with respect to $\left\{e_{i}\right\}$ and $\left\{e_{a}\right\}$. The equations (3.8) and (3.10) are called respectively the equations of Gauss and those of Ricci for the submanifold $M$. The forms $\left(\omega_{j}^{i}\right)$ define the Riemannian connection of $M$ and the forms $\left(\omega_{b}^{a}\right)$ define the connection induced in the normal bundle of $M$.

We now state a lemma for later use.

LEMMA 3.1. (Yano and Kon [6]) Let $M$ be an $(n+1)$-dimensional ant2-invariant submanifold of a Sasakian manifold $\bar{M}^{2 m+1}$. Then the structure vector field $\xi$ is parallel along $M$ and $M$ is locally a Riemannian direct product $M^{n} \times M^{1}$, where 
$M^{n}$ is an n-dimensional Riemannian manrfold and $M^{1}$ is a 1-dimensional subspace generated by $\xi$.

From (3.3), (3.4) and (3.5) we have along $M$

$$
h_{y z}^{x}=h_{x z}^{y}=h_{x y}^{z}, \quad h_{0 i}^{\lambda}=0, \quad h_{0 i}^{x}=\delta_{x \imath},
$$

where we denote $h_{\imath \jmath}^{x^{*}}$ simply by $h_{\imath \jmath}^{x}$.

The second fundamental form $h_{\imath j}^{a} \omega^{2} \omega^{j} e_{a}$ is sometimes denoted by its components $h_{\imath \jmath}^{a}$. When the second fundamental form vanishes identically, i. e., $h_{\imath j}^{a}=0$ for all indices, the submanifold is as usual said to be totally geodesic. If $h_{x y}^{a}$ have the form $h_{c y}^{a}=\frac{1}{n}\left(\sum_{z} h_{z z}^{a}\right) \delta_{x y}$ for a fixed index $a$, then the submanifold is said to be contact umbilical with respect to the normal vector $e_{a}$. If the submanifold $M$ is contact umbilical with respect to all $e_{a}$, then $M$ is said to be contact totally umbilical (see [2]). The vector field $\frac{1}{n+1}\left(\sum_{k} h_{k k}^{a} e_{a}\right)$ normal to $M$ is called the mean curvature vector of $M$. The submanifold $M$ is said to be minımal if its mean curvature vector vanishes identically, i.e., $\sum_{k} h_{k k}^{a}=0$ for all $a$. We define the covariant derivative $h_{\imath j k}^{a}$ of $h_{\imath \jmath}^{a}$ by

$$
h_{\imath j k}^{a} \omega^{k}=d h_{\imath \jmath}^{a}-h_{\imath l}^{a} \omega_{j}^{l}-h_{\imath \jmath}^{a} \omega_{i}^{l}+h_{\imath j}^{b} \omega_{b}^{a} .
$$

If $h_{\imath j k}^{a}=0$ for all indices, the second fundamental form of $M$ is said to be parallel. If the mean curvature vector of $M$ is parallel with respect to the connection $D$ induced in the normal bundle, then the mean curvature vector of $M$ is said to be parallel. The Laplacian $\Delta h_{\imath \jmath}^{a}$ of $h_{\imath \jmath}^{a}$ is defined as

$$
\Delta h_{\imath j}^{a}=\sum_{k} h_{\imath j k k}^{a},
$$

where we have defined $h_{\imath j k l}^{a}$ by

$$
h_{\imath \jmath k l}^{a} \omega^{l}=d h_{\imath j k}^{a}-h_{l \ell k}^{a} \omega_{\imath}^{l}-h_{\iota l k}^{a} \omega_{j}^{l}-h_{\imath j l}^{a} \omega_{k}^{l}+h_{\imath j k}^{b} \omega_{0}^{a} .
$$

We shall establish a formula containing the Laplacian of $h_{\imath \jmath}^{a}$. Now, the second fundamental form of $M$ is assumed to satisfy equations of Codazzi type, i. e.,

$$
h_{\imath j k}^{a}-h_{\iota k J}^{a}=0 .
$$

Then, from (3.14), we have

$$
h_{\imath j k l}^{a}-h_{\imath j l k}^{a}=h_{\imath t}^{a} R_{j k l}^{t}+h_{t j}^{a} R_{\imath k l}^{t}-h_{\imath j}^{b} R_{b k l}^{a} .
$$

On the other hand, (3.13) and (3.15) imply

$$
\Delta h_{\imath j}^{a}=\sum_{k} h_{\imath j k k}^{a}=\sum_{k} h_{k \imath j k}^{a} .
$$


From (3.15), (3.16) and (3.17), we obtain

$$
\Delta h_{\imath \jmath}^{a}=\sum_{k}\left(h_{k k \imath \jmath}^{a}+h_{k t}^{a} R_{\imath j k}^{t}+h_{t \imath}^{a} R_{k \jmath k}^{t}-h_{k \imath}^{b} R_{b j k}^{a}\right) .
$$

Therefore for any submanifold $M$ satisfying the equation (3.15) of Codazzi type we have the formula

$$
\begin{aligned}
\sum_{a, \imath, \jmath} h_{\imath j}^{a} \Delta h_{\imath \jmath}^{a}= & \sum_{a, \imath, j, k}\left(h_{\imath j}^{a} h_{k k \imath \jmath}^{a}+h_{\imath j}^{a} h_{k t}^{a} R_{\imath j k}^{t}\right. \\
& \left.+h_{\imath j}^{a} h_{t \imath}^{a} R_{k j k}^{t}-h_{\imath j}^{a} h_{k \imath}^{b} R_{b j k}^{a}\right) .
\end{aligned}
$$

We are now going to prove some lemmas for later use. From (3.3), (3.11) and (3.12), we have

$$
\begin{gathered}
h_{\imath \jmath 0}^{\alpha}=-h_{\imath \jmath}^{\alpha^{*}}, \quad h_{\imath j 0}^{\alpha^{*}}=h_{\imath \jmath}^{\alpha}, \quad h_{\imath j 0}^{x}=0, \\
\sum_{a, \imath, \jmath, k}\left(h_{\imath j k}^{a}\right)^{2}=\sum_{a, x, y, z}\left(h_{x y z}^{a}\right)^{2}+3 \sum_{\lambda, x, y}\left(h_{x y}^{\lambda}\right)^{2}, \\
\sum_{a, \imath, \jmath, k} h_{\imath j}^{a} h_{k k \imath j}^{a}=\sum_{a, x, j, k} h_{x j}^{a} h_{k k x \jmath}^{a}-\sum_{\lambda}\left(\sum_{k} h_{k k}^{\lambda}\right)^{2} .
\end{gathered}
$$

Thus, we have from (3.20)

LEMMA 3.2. Let $M$ be an $(n+1)$-dimensional anti-invariant submanfold of a Sasakian manifold $\bar{M}^{2 m+1}$. If the second fundamental form of $M$ is parallel, then $h_{\imath \jmath}^{\lambda}=0$ for all $\lambda$.

Using (3.20), we obtain

$$
\sum_{\imath} h_{i i 0}^{\alpha}=-\sum_{\imath} h_{\imath \imath}^{\alpha^{*}}, \quad \sum_{i} h_{i i 0}^{\alpha^{*}}=\sum_{\imath} h_{\imath i}^{\alpha},
$$

which imply

LEMMA 3.3. Let $M$ be an $(n+1)$-dimensional anti-invariant submanfold of a Sasakian manifold $\bar{M}^{2 m+1}$. If the mean curvature vector of $M$ is parallel, then $\sum_{\imath} h_{\imath i}^{\lambda}=0$ for all $\lambda$.

When $m>n$, Lemmas 3.2 and 3.3 show that the conditions that the second fundamental form is parallel and that the mean curvature vector is parallel are not meaningful for anti-invariant submanifolds. Therefore we shall now introduce some new concepts as follows. On an anti-invariant submanifold $M$ of a Sasakian manifold $\bar{M}^{2 m+1}$, if $h_{x y z}^{a}=0$ for all indices $a, x, y$ and $z$, then we say that the second fundamental form of $M$ is pseudo-parallel. If $\sum_{\imath} h_{i i x}^{a}=0$ for all indices $a$ and $x$, then the mean curvature vector said to be pseudo-parallel.

If the ambient manifold $\bar{M}^{2 m+1}$ is of constant $\phi$-sectional curvature $c$, then the Riemannian curvature tensor of $\bar{M}^{2 m+1}$ has the form

$$
K_{B C D}^{A}=\frac{1}{4}(c+3)\left(\delta_{A C} \delta_{B D}-\delta_{A D} \delta_{B C}\right)+\frac{1}{4}(c-1)\left(\eta_{B} \eta_{C} \delta_{A D}\right.
$$




$$
\begin{aligned}
& -\eta_{B} \eta_{D} \delta_{A C}+\eta_{A} \eta_{D} \delta_{B C}-\eta_{A} \eta_{C} \delta_{B D}+\phi_{A C} \phi_{B D} \\
& \left.-\phi_{A D} \phi_{B C}+2 \phi_{A B} \phi_{C D}\right),
\end{aligned}
$$

and the second fundamental form of $M$ satisfies the equation (3.15) of Codazzi type.

\section{$\S 4$. The connection $D$ in the normal bundle.}

In this section we study the connection $D$ induced in the normal bundle to an $(n+1)$-dimensional anti-invariant submanifold $M$ of a $(2 m+1)$-dimensional Sasakian space form $\bar{M}^{2 m+1}$ (c) when the structure vector field $\xi$ is tangent to $M$.

First of all, by (3.24) we obtain

$$
\begin{aligned}
& K_{x^{*} k l}^{\lambda}=0, \quad K_{j k l}^{\lambda}=0, \quad K_{\mu k l}^{\lambda}=0, \\
& K_{y^{*} k l}^{x^{*}}=\frac{1}{4}(c-1)\left(\delta_{x k} \delta_{y l}-\delta_{x l} \delta_{y k}\right) .
\end{aligned}
$$

From (3.10), (3.11) and (4.1), we have

$$
R_{x^{*} y 0}^{\lambda}=h_{x y}^{\lambda}, \quad R_{y^{*} z 0}^{x^{*}}=0 .
$$

The connection $D$ induced in the normal bundle to $M$ is said to be flat if $R_{b_{1}}^{a}=0$ for all indices. Thus we have from (3.11) and (4.2)

LEMMA 4.1. Let $M$ be an $(n+1)$-demensional anti-invariant submanifold of a Sassakian space form $\bar{M}^{2 m+1}(c)$. If the connection $D$ is flat then $h_{i j}^{\lambda}=0$.

When $m>n$, Lemma 4.1 shows that the condition that the connection $D$ is flat is not meaningful for anti-invariant submanifolds of a Sasakian space form. Therefore the pseudo-flatness of $D$ will be introduced as follows. On an antiinvariant submanifold $M$ of a Sasakian manifold $\bar{M}^{2 m+1}$ the connection $D$ is said to be pseudo-flat if $R_{b x y}^{a}=0$ for all indices.

LEMMA 4.2. Let $M$ be an $(n+1)$-dimensional anti-invariant submanfold of a Sasakian manifold $\bar{M}^{2 m+1}$. If the connection $D$ is pseudo-fiat then

$$
R_{y z w}^{x}=\sum_{\lambda}\left(h_{x z}^{\lambda} h_{y w}^{\lambda}-h_{x w}^{\lambda} h_{y z}^{\lambda}\right) .
$$

Proof. (3.2) and (3.3) imply

$$
K_{y^{*} z}^{x^{*}}=K_{y z w}^{x}-\left(\delta_{x z} \delta_{y w}-\delta_{x w} \delta_{y z}\right) .
$$

Moreover, from (3.8), (3.10) and (3.11), we obtain

$$
\begin{aligned}
R_{y z w}^{x} & =K_{y z w}^{x}+\sum_{a}\left(h_{x z z}^{a} h_{y w}^{a}-h_{x w}^{a} h_{y z}^{a}\right) \\
& =K_{y z w}^{x}-\left(\delta_{x z} \delta_{y w}-\delta_{x w} \delta_{y z}\right)+\sum_{t}\left(h_{t z}^{x} h_{t w}^{y}-h_{t u}^{x} \cdot h_{t z}^{y}\right)
\end{aligned}
$$




$$
\begin{aligned}
& +\sum_{\lambda}\left(h_{x z}^{\lambda} h_{y w}^{\lambda}-h_{x w}^{\lambda} h_{y z}^{\lambda}\right) \\
= & R_{y^{*} z}^{x^{*}}+\sum_{\lambda}\left(h_{x z}^{\lambda} h_{y w}^{\lambda}-h_{x w}^{\lambda} h_{y z}^{\lambda}\right) .
\end{aligned}
$$

This completes the proof.

From (3.3) and (3.8), we have

$$
R_{\imath j k}^{0}=0 \text {. }
$$

Thus Lemmas 4.1 and 4.2 imply immediately

COROLlARy 4.3. Let $M$ be an $(n+1)$-dimensional antı-invariant submanifold of a Sasakian space form $\bar{M}^{2 m+1}(c)$. If the connection $D$ is flat, then $M$ is flat.

If the connection $D$ is pseudo-flat, then (3.10) and (4.1) imply

$$
\begin{gathered}
\sum_{\imath}\left(h_{\imath x}^{\lambda} h_{\imath y}^{v}-h_{\imath y}^{\lambda} h_{\imath x}^{v}\right)=0, \quad \sum_{\imath}\left(h_{\imath x}^{\lambda} h_{\imath y}^{\mu}-h_{\imath y}^{\lambda} h_{\imath x}^{\mu}\right)=0, \\
\sum_{\imath}\left(h_{\imath x}^{v} h_{\imath y}^{w}-h_{\imath y}^{v} h_{\imath x}^{w}\right)=-\frac{1}{4}(c-1)\left(\delta_{v x} \delta_{w y}-\delta_{v y} \delta_{w x}\right) .
\end{gathered}
$$

Moreover, using (3.11), we have

$$
\begin{aligned}
& \sum_{z}\left(h_{z x}^{\lambda} h_{z y}^{v}-h_{z y}^{\lambda} h_{z x}^{v}\right)=0, \quad \sum_{z}\left(h_{z x}^{\lambda} h_{z y}^{\mu}-h_{z y}^{\lambda} h_{z x}^{\mu}\right)=0, \\
& \sum_{z}\left(h_{z x}^{v} h_{z y}^{w}-h_{z y}^{v} h_{z x}^{w}\right)=-\frac{1}{4}(c+3)\left(\delta_{v x} \delta_{w y}-\delta_{v y} \delta_{w x}\right) .
\end{aligned}
$$

Proposition 4.4. Let $M$ be an $(n+1)$-dimensional $(n>1)$ anti-invariant submanifold of a Sasakian space form $\bar{M}^{2 m+1}(c)$ and the connection $D$ induced in the normal bundle to $M$ be pseudo-flat. Then, if $M$ is contact umbilical with respect to $e_{v^{*}}$ for some index $v$, then $c=-3$.

Proof. If $M$ is contact umbilical with respect to $e_{v^{*}}$, then $h_{x y}^{v}$ is of the form $h_{x y}^{v}=\frac{1}{n}\left(\sum_{z} h_{z z}^{v}\right) \delta_{x y}$. Moreover, using (3.11), we have

$$
\sum_{\imath}\left(h_{\imath x}^{v} h_{\imath y}^{w}-h_{\imath y}^{v} h_{\imath x}^{w}\right)=\delta_{v x} \delta_{w y}-\delta_{v y} \delta_{w x} .
$$

From this and (4.8) we find $c=-3$.

For each index $a$, the second fundamental form will be represented by a symmetric $(n+1, n+1)$-matrix $A_{a}=\left(h_{\imath j}^{a}\right)$ composed of its components. Following such notations, we have from (3.11)

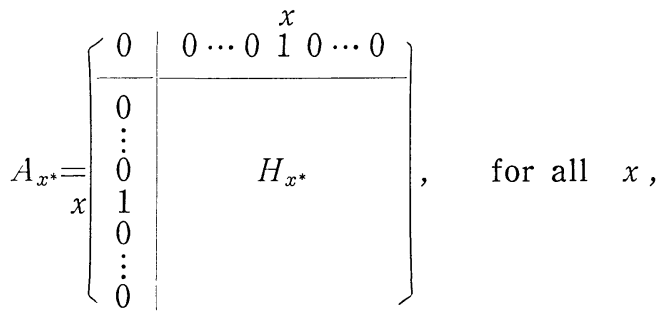




$$
A_{\lambda}=\left(\begin{array}{c|c}
0 & 0 \cdots \cdots \cdots 0 \\
\hline 0 & \\
\vdots & H_{\lambda} \\
\vdots &
\end{array}\right], \quad \text { for all } \lambda,
$$

where $H_{a}=\left(h_{x y}^{a}\right)$ are symmetric $(n, n)$-matrices.

LEMMA 4.5. Let $M$ be an $(n+1)$-dimensional antr-invariant submanfold of a Sasakian space form $\bar{M}^{2 m+1}(c)(c \neq-3)$. If the connection $D$ is pseudo-flat, then $M$ is contact umbilical with respect to all $e_{\lambda}$.

Proof. From (4.9) we obtain $H_{\lambda} H_{\mu}=H_{\mu} H_{\lambda}$ and $H_{\lambda} H_{1}=H_{1} H_{\lambda}$ for all $\lambda$ and $\mu$. Therefore we can choose a local field of orthonormal frames with respect to which $H_{1}$ and all $H_{\lambda}$ are simultaneously diagonal i.e.,

$$
A_{1}=\left(\begin{array}{c|cccc}
0 & 1 & 0 & \cdots & 0 \\
\hline 1 & h_{11}^{1} & & \\
0 & & & \\
\vdots & & & \cdot \\
0 & & & h_{n n}^{1}
\end{array}\right), \quad A_{\lambda}=\left(\begin{array}{c|ccc}
0 & 0 & \cdots & 0 \\
\hline 0 & h_{11}^{\lambda} & & \\
\vdots & & \cdot & \\
0 & & & \cdot \\
h_{n n}^{\lambda}
\end{array}\right) .
$$

Putting $x=1$ and $v=y$ in the first equation of (4.9) and using (3.11) and (4.11), we find

$$
\left(h_{11}^{\lambda}-h_{y y}^{\lambda}\right) h_{y y}^{1}=0 .
$$

On the other hand, putting $v=x=1$ and $w=y \neq 1$ in (4.10) and using (3.11) and (4.11), we have

$$
\left(h_{11}^{1}-h_{y y}^{1}\right) h_{y y}^{1}=-\frac{1}{4}(c+3) .
$$

Since $c \neq-3,(4.13)$ implies $h_{y y}^{1} \neq 0(y=2, \cdots, n)$. From this fact and (4.12) we find that $h_{11}^{\lambda}=h_{y y}^{\lambda}$ for all $\lambda$. Thus $M$ is contact umbilical with respect to all $e_{\lambda}$. This proves Lemma 4.5 .

LEMMA 4.6. Let $M$ be an $(n+1)$-dimensional antr-invariant submanifold of a Sasakian space form $\bar{M}^{2 m+1}(c)(c \neq-3)$. If the connection $D$ is pseudo-flat, then

$$
R_{y z w}^{x}=\frac{1}{n^{2}} \sum_{\lambda}\left(\operatorname{Tr} A_{\lambda}\right)^{2}\left(\delta_{x z} \delta_{y w}-\delta_{x w} \delta_{y z}\right) .
$$

Proof. Lemma 4.5 implies $h_{x y}^{\lambda}=\frac{1}{n}\left(\operatorname{Tr} A_{\lambda}\right) \delta_{x y}$ for all $\lambda$. Therefore (4.3) implies (4.14).

Proposition 4.7. Let $M$ be an $(n+1)$-dimensional ( $n \geqq 3)$ anti-invariant submanıfold of a Sasakıan space form $\bar{M}^{2 m+1}(c)(c \neq-3)$. If the connection $D$ induced 
in the normal bundle to $M$ is pseudo-flat, then $M$ is locally a Riemannian direct product $M^{n} \times M^{1}$, where $M^{n}$ is of constant curvature, and $M^{1}$ is a 1-dimensional subspace generated by $\xi$.

Proof. Since $n \geqq 3$, (4.14) implies that $\sum_{\lambda}\left(\operatorname{Tr} A_{\lambda}\right)^{2}$ is constant. Therefore Proposition 4.7 is proved by means of Lemma 3.1 .

If $M$ is minimal, then $\operatorname{Tr} A_{\lambda}=0$ for all $\lambda$. Thus Lemma 4.5 and (4.2) imply immediately

Proposition 4.8. Let $M$ be an $(n+1)$-dimensional antı-ınvariant minımal submanifold of a Sasakian space form $\bar{M}^{2 m+1}(c)(c \neq-3)$. Then the connection $D$ induced in the normal bundle to $M$ is flat if and only if the connection $D$ is pseudoflat.

Moreover, Corollary 4.3 and Proposition 4.8 imply immediately

Corollary 4.9. Let $M$ be an $(n+1)$-dimensional antr-invarrant minimal submanifold of a Sasakian space form $\bar{M}^{2 m+1}(c)(c \neq-3)$. If the connection $D$ induced in the normal bundle to $M$ is pseudo-flat, then $M$ is flat.

\section{$\S 5$. Pseudo-parallel mean curvature vector.}

Using the results obtain in the previous section, we have

THEOREM 1. Let $M$ be an $(n+1)$-dimensional $(n \geqq 3)$ antı-invariant submanıfold of a Sasakian space form $\bar{M}^{2 m+1}(c)(c \neq-3)$ with pseudo-parallel mean curvature vector. If the connection $D$ induced in the normal bundle to $M$ is pseudo-flat, then there is in $\bar{M}^{2 m+1}(c)$ a totally geodesic and invariant submanifold $\bar{M}^{2 n+1}(c)$ of dimension $2 n+1$ in such a way that $M$ is immersed $2 n \bar{M}^{2 n+1}(c)$ as a flat antrinvariant submanfold.

Proof. (3.23) implies that $\sum_{a}\left(\operatorname{Tr} A_{a}\right)^{2}$ is constant because the mean curvature vector is pseudo-parallel. Since $n \geqq 3$, (4.14) implies that $\sum_{\lambda}\left(\operatorname{Tr} A_{\lambda}\right)^{2}$ is constant. On the other hand, from (3.8), (3.24) and (4.14), we have

$$
\frac{n-1}{n} \sum_{\lambda}\left(\operatorname{Tr} A_{\lambda}\right)^{2}=\frac{1}{4} n(n-1)(c+3)+\sum_{a}\left(\operatorname{Tr} A_{a}\right)^{2}-\sum_{a, x, y}\left(h_{x y}^{a}\right)^{2} .
$$

Moreover, using (3.11), we find that the square of the length of the second fundamental form of $M$ is constant, i.e., $\sum_{a, 2, j}\left(h_{\imath j}^{a}\right)^{2}$ is constaant. From this we have

$$
\sum_{a, i, j, k}\left(h_{\imath j k}^{a}\right)^{2}+\sum_{a, \imath, j} h_{\imath j}^{a} \Delta h_{\imath \jmath}^{a}=\frac{1}{2} \Delta \sum_{a, \imath, j}\left(h_{i j}^{a}\right)^{2}=0 .
$$

On the other hand the mean curvature vector is pseudo-parallel and the connection $D$ is pseudo-flat. Thus (3.11), (3.19), (3.22) and (4.6) imply 


$$
\begin{aligned}
\sum_{a, 2, j} h_{\imath, j}^{a} \Delta h_{\imath, j}^{a}= & -\sum_{\lambda}\left(\operatorname{Tr} A_{\lambda}\right)^{2}+\sum_{a, x, y, z, w} h_{y z}^{a} h_{w, x}^{a} R_{y z w}^{x} \\
& +\sum_{a, \imath, x, y, z} h_{\iota z}^{a} h_{x \imath}^{a} R_{y z y}^{x}-2 \sum_{a, x, y} h_{x y}^{a} R_{x^{*} y 0}^{a} .
\end{aligned}
$$

Moreover, substituting (4.14) into (5.3), we obtain by using (3.11) and (5.2),

$$
\begin{aligned}
\sum_{a, \imath, j, k}\left(h_{\imath, k}^{a}\right)^{2}= & -\frac{1}{n^{2}} \sum_{\lambda}\left(\operatorname{Tr} A_{\lambda}\right)^{2}\left(\sum_{a, x, y}\left(n\left(h_{, x y}^{a}\right)^{2}-h_{x, x}^{a} h_{y y}^{a}\right)-n\right) \\
& +2 \sum_{\lambda, x, y}\left(h_{x y y}^{\lambda}\right)^{2}
\end{aligned}
$$

from which

$$
\begin{aligned}
\sum_{a, y, z}\left(h_{x y z}^{a}\right)^{2} & =-\frac{1}{n^{2}} \sum_{\lambda}\left(\operatorname{Tr} A_{\lambda}\right)^{2}\left(\sum_{x, y, z}\left(n\left(h_{x y}^{z}\right)^{2}-h_{x x}^{z} h_{y y}^{z}\right)\right) \\
& =-\frac{1}{n^{2}} \sum_{\lambda}\left(\operatorname{Tr} A_{\lambda}\right)^{2} \sum_{z}\left(\sum_{x<y}\left(h_{x x}^{z}-h_{y y}^{z}\right)^{2}+\sum_{x \neq y}\left(h_{x y}^{z}\right)^{2}\right),
\end{aligned}
$$

by means of Lemma 4.5 and (3.21).

Since $c \neq-3$ by assumption, Proposition 4.4 implies $\sum_{x<y}\left(h_{x x}^{z}-h_{y y}^{z}\right)^{2}>0$. Thus (5.5) implies $\operatorname{Tr} A_{\lambda}=0$ for all $\lambda$ which mean that $A_{\lambda}=0$ for all $\lambda$. And from (5.5) we see that $h_{x^{\prime} y z}^{a}=0$, i. e., that the second fundamental form of $M$ is pseudoparallel. Since $A_{\lambda}=0$ for all $\lambda$, (3.20) implies $h_{\iota \jmath 0}^{a}=0$. Therefore the second fundamental form of $M$ is parallel. Moreover, since $A_{\lambda}=0$ for all $\lambda$, Lemma 4.6 and (4.6) imply that $M$ is flat. From the arguments above, taking account of a fundamental theorem in the theory of submanifolds, we see that $M$ is an antiinvariant submanifold immersed in some totally geodesic and $(2 n+1)$-dimensional submanifold $\bar{M}^{2 n+1}(c)$ of $\bar{M}^{2 m+1}(c)$ (see $\S 6$ ). And the submanifold $\bar{M}^{2 n+1}(c)$ is invariant (see $\$ 6$ ). Thus Theorem 1 is proved.

In Theorem 1, the case where $n=2$, that is, where $M$ is 3-dimensional, is excluded. However, the same conclusions will be established even if $n=2$, provided that $M$ is compact. To establish this fact, we now prove

THEOREM 2. Let $M$ be a compact $(n+1)$-dimensional $(n>2)$ antl-invariant submanifold of a Sasakian space form $\bar{M}^{2 m+1}(c)(c \neq-3)$ with pseudo-parallel mean curvature vector. If the connection $D$ induced in the normal bundle to $M$ is pseudoflat, then there is in $\bar{M}^{2 m+1}(c)$ a totally geodesic and invariant submanifold $\bar{M}^{2 n+1}(c)$ of dimension $2 n+1$ in such a way that $M$ is immersed $\imath n \bar{M}^{2 n+1}(c)$ as a flat antrinvariant submanifold.

Proof. Since $M$ is compact, we have

$$
\int_{M} \sum_{a, i, j, k}\left(h_{\imath j k}^{a}\right)^{2 * 1} 1=-\int_{M} \sum_{a, \imath, j} h_{i j}^{a} \Delta h_{\imath \jmath}^{a} * 1,
$$

where $* 1$ denotes the volume element of $M$. Using this formula, we can prove Theorem 2 by a same way as taken to prove Theorem 1 . 
In the proof of Theorem 1, the following Corollary 5.1 has already been proved.

Corollary 5.1. Let $M$ be an $(n+1)$-dimensional $(n \geqq 3)$ antr-invarzant submanifold of a Sasakian space form $\bar{M}^{2 m+1}(c)(c \neq-3)$ with pseudo-flat normal connection. Then following notions $(a),(b),(c),(d)$ are equivalent to each other: $(a)$ the mean curvature vector is pseudo-parallel, (b) the mean curvature vector is parallel, (c) the second fundamental form is pseudo-parallel, $(d)$ the second fundamental form is parallel.

Theorem 1 and (4.2) imply immediatelly

COROLlary 5.2. Let $M$ be an $(n+1)$-dimensional $(n \geqq 3)$ antr-invariant submanifold of a Sasakian space form $\bar{M}^{2 m+1}(c)(c \neq-3)$ with pseudo-parallel mean curvature vector. Then the connection $D$ induced in the normal bundle to $M$ is flat if and only if the connection $D$ is pseudo-flat.

Remark. By Theorem 2, if $M$ is compact, Corollaries 5.1 and 5.2 hold even for $n=2$.

We shall now study submanifolds of a Sasakian space form $\bar{M}^{2 m+1}(c)$ in the case where $c=-3$.

Proposition 5.3. Let $M$ be an $(n+1)$-dimensional $(n \geqq 3)$ antl-invariant submanifold of a Sasakian space form $\bar{M}^{2 m+1}(-3)$ with pseudo-parallel mean curvature vector and with pseudo-flat normal connection. If $M$ is contact umbilical with respect to all $e_{\lambda}$, then $M$ is either a flat anti-invariant submanifold immersed in some totally geodesic and $(2 n+1)$-dimensional subman fold $\bar{M}^{2 n+1}(-3)$ of $\bar{M}^{2 m+1}(-3)$, or a totally contact umbilical submanifold.

Proof. From the assumption and Lemma 4.2 we have the equation (4.14). Therefore the equation (5.5) holds. Thus we have either $\operatorname{Tr} A_{\lambda}=0$ for all $\lambda$, or $\sum_{z}\left(\sum_{x<y}\left(h_{x x}^{z}-h_{y y}^{z}\right)^{2}+n \sum_{x \neq y}\left(h_{x y}^{z}\right)^{2}\right)=0$. In this step, we can prove the following fact by the same way as taken to prove Theorem $1: M$ is flat and immersed in some totally geodesic and $(2 n+1)$-dimensional submanifold $\bar{M}^{2 n+1}(-3)$ of $\bar{M}^{2 m+1}$ $(-3)$ as an anti-invariant submanifold, when $\operatorname{Tr} A_{\lambda}=0$ for all $\lambda$. When $\sum_{z}\left(\sum_{x<y}\left(h_{x x}^{z}-h_{y y}^{z}\right)^{2}+n \sum_{x \neq y}\left(h_{x y}^{z}\right)^{2}\right)=0, h_{x x}^{z}=h_{y y}^{z}, h_{x y}^{z}=0(x \neq y)$ hold and hence $M$ is contact umbilical with respect to each $e_{z^{*}}$. Therefore, in this cace, $M$ is a totally contact umbilical. Thus Proposition 5.3 is proved completely.

Remark. If in Proposition $5.3 M$ is totally contact umbilical, then (3.11) implies $H_{z}=0$ for all $z$.

THEOREM 3. Let $M$ be an $(n+1)$-dimensional antz-invariant submanifold of a Sasakian space form $\bar{M}^{2 m+1}(c)$. If the second fundamental form is parallel, then $M$ is an antr-invariant submanifold immersed in some totally geodesic and $(2 n+1)$ dimensional submanifold $\bar{M}^{2 n+1}(c)$ of $\bar{M}^{2 m+1}(c)$. 
Proof. From Lemma 3.2, (3.3) and (3.5) we have $\omega_{x^{*}}^{\lambda}=-\omega_{x}^{\lambda}=-h_{x i}^{\lambda} \omega^{2}=0$, which and (3.12) imply $h_{\imath j k}^{\lambda}=0$. From these and a fundamental theorem in the theory of submanifolds, we have our assertion.

Using Lemma 4.1, we have the following Theorem 4 from Corollary 4.3 by the same way as taken in the proof of Theorem 3.

THEOREM 4. Let $M$ be an $(n+1)$-dimensional anti-invariant submanifold of a Sasakian space form $\bar{M}^{2 m+1}(c)$. If the connection $D$ induced in the normal bundle to $M$ is flat, then $M$ is a flat ant-invariant submanifold immersed in some totally geodesic and $(2 n+1)$-dimensional submanifold $\bar{M}^{2 n+1}(c)$ of $\bar{M}^{2 m+1}(c)$.

According to a theorem due to Yano and Kon (see [7], p. 100), Theorem 2 and Corollaries 5.1, 5.2 imply

THEOREM 5. Let $M$ be an $(n+1)$-dimensional $(n>2)$ compact orientable antrinvariant submanifold with pseudo-parallel mean curvature vector of $S^{2 m+1}$. If the connection $D$ induced in the normal bundle to $M$ is pseudo-flat, then

$$
M=S^{1}\left(r_{1}\right) \times \cdots \times S^{1}\left(r_{n+1}\right)
$$

immersed in an $S^{2 n+1}$ which is totally geodesic in $S^{2 m+1}$, where $r_{1}^{2}+\cdots+r_{n+1}^{2}=1$.

\section{$\S 6$. Axiom of $\phi$-holomorphic planes.}

Let $M$ be a $(2 n+1)$-dimensional Sasakian manifold with structure tensors $(\phi, \xi, \eta, g)$ covered by a system of coordinate neighborhoods $\left\{U, x^{i}\right\}$. (In this section, indices $i, \jmath, k, l, p, q, r, s, t$ run over the range $\{1, \cdots, 2 n+1\}$ and the summation convention is used with respect to this system of indices.)

We assume that a Sasakian manifold $M$ admits the axiom of $\phi$-holomorphic $(2 r+1)$-planes; that is, for each point $p$ of $M$ and any $(2 r+1)$-dimensional $\phi$ holomorphic subspace $S$ of $T_{p}(M), 1<r<n$, there exists a $(2 r+1)$-dimensional totally geodesic submanifold $N$ passing through $p$ and satisfying $T_{p}(N)=S$, where we mean a $\phi$-holomorphic subspace $S$ by a subspace of $T_{p}(M)$ satisfying $\phi S \subset S$. Since rank $\phi$ is $2 n$, the subspace $S$ contains the structure vector field $\xi$ of the ambient manifold. Thus, by assumption, there is a $(2 r+1)$-dimensional totally geodesic submanifold $N$ passing through this point $p$ and being tangent to given subspace $S$. If we represent such a submanifold $N$ by parametric equations

$$
x^{2}=x^{2}\left(y^{a}\right),
$$

where $\left\{y^{a}\right\}$ are local coordinates in $N$, (In this section, the indices $a, b, c, d$ run over the range $\{1, \cdots, 2 r+1\}$ and the summation convention is used with respect to this system of indices.) then the fact that the submanifold $N$ is totally geodesic is represented by the equations 


$$
\frac{\partial^{2} x^{2}}{\partial y^{a} \partial y^{b}}+\left\{\begin{array}{c}
i \\
j k
\end{array}\right\} \frac{\partial x^{j}}{\partial y^{a}}-\frac{\partial x^{k}}{\partial y^{b}}-\left\{\begin{array}{c}
c \\
a b
\end{array}\right\} \frac{\partial x^{\imath}}{\partial y^{c}}=0,
$$

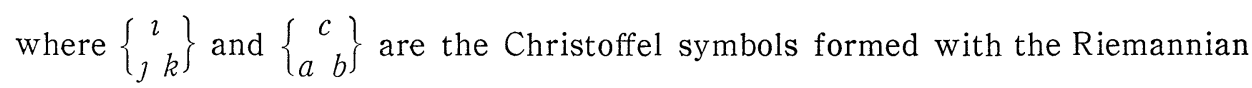
metric tensor $g_{\imath}$ of the ambient manifold $M$ and the naturally induced Riemannian metric tensor $g_{a b}=g_{\imath} \frac{\partial x^{\imath}}{\partial y^{a}}-\frac{\partial x^{\jmath}}{\partial y^{b}}$ of the submanifold $N$ respectively.

The integrability condition of the differential equation (6.2) is

$$
B_{c}{ }^{k} B_{d}{ }^{l} B_{b}{ }^{\jmath} K_{j k l}^{i}=B_{a}{ }^{\imath} R_{b c d}^{q},
$$

where $K_{j k l}^{i}$ and $R_{b c d}^{a}$ are the curvature tensors of the ambient manifold $M$ and the submanifold $N$ respectively, and $B_{a}{ }^{2}=\partial x^{2} / \partial y^{a}$.

We first assume that $M$ satisfies the axiom of $\phi$-holomorphic 3-planes. Take arbitrarily a unit vector field $v$ tangent to $N$. If we put $B_{1}=\phi v, B_{2}=\phi^{2} v$, then we have by (6.3)

$$
\left(\phi_{s}{ }^{t} v^{s}\right)\left(\phi_{q}{ }^{r} \phi_{l}{ }^{q} v^{l}\right)\left(\phi_{p}{ }^{k} \phi_{\jmath}{ }^{p} v^{j}\right) K_{k t r}^{i}=\alpha \phi_{\jmath}{ }^{\imath} v^{j}+\beta \phi_{q}{ }^{\imath} \phi_{\jmath}{ }^{q} v^{\jmath}+\gamma \xi^{\imath},
$$

from which

$$
\left(\phi_{s}{ }^{t} K_{j t l}^{i}-\phi_{s}{ }^{2} \eta_{l} \eta_{\jmath}\right) v^{s} v^{l} v^{\jmath}=\left(\alpha \phi_{\jmath}{ }^{2}-\beta \delta_{\jmath}{ }^{2}+\beta \xi^{\imath} \eta_{\jmath}\right) v^{\jmath}+\gamma \xi^{2},
$$

or equivalently

$$
\left(\phi_{s}{ }^{t} K_{\imath \jmath t l}-\phi_{s i} \eta_{l} \eta_{j}\right) v^{s} v^{l} v^{j}=\left(\alpha \phi_{j \imath}-\beta g_{j i}+\beta \eta_{\imath} \eta_{j}\right) v^{j}+\gamma \eta_{\imath},
$$

where $K_{\imath \jmath t l}=K_{j t l}^{k} g_{\imath k}$ and $\phi_{s i}=\dot{\phi}_{s}{ }^{k} g_{\imath k}$. Transvecting $(6.5)^{\prime}$ with $\xi^{\imath}$, we get $\gamma=0$. On the other hand, transvecting $(6.5)^{\prime}$ with $v^{2}$, we obtain $\beta=0$, because $K_{\imath j t l}$ and $\phi_{i j}$ are skew-symmetric with respect to $\iota$ and $\jmath$.

If we put

$$
c=\frac{\alpha}{\|\phi v\|^{2}}=\frac{\alpha}{\left(g_{s l}-\eta_{s} \eta_{l}\right) v^{s} v^{l}},
$$

then we have from (6.5)

$$
\left(\phi_{s}{ }^{t} K_{l t l}^{i}-\phi_{s}{ }^{2} \eta_{l} \eta_{j}\right) v^{s} v^{l} v^{\jmath}=c\left(g_{s l}-\eta_{s} \eta_{l}\right) v^{s} v^{l} v^{j},
$$

from which

$$
\begin{aligned}
\phi_{s}{ }^{t} K_{j t l}^{i} & +\phi_{s}{ }^{t} K_{l t \jmath}^{i}+\phi_{l}{ }^{t} K_{s t \jmath}^{i}+\phi_{l}{ }^{t} K_{j t s}^{i}+\phi_{\jmath}{ }^{t} K_{l t s}^{i}+\phi_{\jmath}{ }^{t} K_{s t l}^{i} \\
& -2\left(\phi_{s}{ }^{2} \eta_{l} \eta_{\jmath}+\phi_{l}{ }^{2} \eta_{j} \eta_{s}+\phi_{\jmath}{ }^{2} \eta_{s} \eta_{l}\right) \\
= & 2 c\left(g_{s l} \phi_{\jmath}{ }^{2}+g_{l j} \phi_{s}{ }^{2}+g_{\jmath s} \phi_{l}{ }^{2}-\eta_{s} \eta_{l} \phi_{\jmath}{ }^{2}-\eta_{l} \eta_{j} \phi_{s}{ }^{2}-\eta_{\jmath} \eta_{s} \phi_{l}{ }^{i}\right) .
\end{aligned}
$$

Transvecting this with $\phi_{k}^{s}$, we obtain

$$
-2 K_{j k l}^{i}-2 K_{l k \jmath}^{i}+\phi_{l}{ }^{t} \phi_{k}{ }^{s} K_{s t \jmath}^{i}+\phi_{\jmath}{ }^{t} \phi_{k}{ }^{s} K_{s t l}^{i}-\delta_{l}{ }^{\imath} g_{k \jmath}-\delta_{\jmath}{ }^{2} g_{k l}
$$




$$
\begin{aligned}
& -2 \hat{\delta}_{k}{ }^{2} g_{l \jmath}+2 g_{j l} \xi^{2} \eta_{k}-\delta_{l}{ }^{2} \eta_{k} \eta_{j}-\delta_{j}{ }^{2} \eta_{k} \eta_{l}+2 \delta_{k}{ }^{2} \eta_{l} \eta_{J} \\
& -2 \xi^{2} \eta_{k} \eta_{l} \eta_{\jmath}+\phi_{l}{ }^{2} \phi_{k \jmath}+\phi_{\jmath}{ }^{2} \phi_{k l} \\
= & 2 c\left(\phi_{k l} \phi_{\jmath}{ }^{2}-g_{l j} \delta_{k}{ }^{2}+g_{l j} \xi^{2} \eta_{k}+\phi_{k j} \phi_{l}{ }^{2}+\delta_{k}{ }^{2} \eta_{l} \eta_{j}-\xi^{2} \eta_{k} \eta_{l} \eta_{\jmath}\right) .
\end{aligned}
$$

On the other hand, we get easily by using the Ricci identity

$$
\begin{aligned}
& \phi_{l}^{t} \dot{\phi}_{k}^{s} K_{s t}^{i}-\phi_{k}{ }^{t} \phi_{l}^{s} K_{s t_{j}}^{i} \\
& =-K_{j k l}^{i}+\phi_{l}{ }^{2} \phi_{k \jmath}-\phi_{k}{ }^{2} \phi_{l \jmath}-\delta_{l}{ }^{2} g_{k \jmath}+\delta_{k}{ }^{2} g_{l \jmath}, \\
& \dot{\phi}_{J}{ }^{t} \phi_{k}{ }^{s} K_{s t l}^{i}-\phi_{J}{ }^{t} \phi_{l}{ }^{s} K_{s t k}{ }^{t} \\
& =-K_{j k l}+2\left(\delta_{k}{ }^{\imath} g_{l \jmath}-\delta_{l}{ }^{\imath} g_{k \jmath}\right)+\delta_{l}{ }^{\imath} \eta_{j} \eta_{k}-\delta_{k}{ }^{2} \eta_{j} \eta_{l}+2 \phi_{k l} \phi_{j}{ }^{2} .
\end{aligned}
$$

Using the equations above and taking the skew-symmetric part with respect to the indices $k$ and $l$ in (6.6), we get

$$
\begin{aligned}
4 K_{j k l}^{i}= & (c+3)\left(\delta_{k}{ }^{2} g_{l j}-\delta_{l}{ }^{2} g_{k j}\right)+(c-1)\left(\delta_{l}{ }^{2} \eta_{k} \eta_{\jmath}-\delta_{k}{ }^{2} \eta_{l} \eta_{\jmath}\right. \\
& \left.+g_{k j} \xi^{2} \eta_{l}-g_{l j} \xi^{2} \eta_{k}-\phi_{k j} \phi_{l}{ }^{2}+\phi_{l j} \phi_{k}{ }^{2}-2 \phi_{k l} \phi_{\jmath}{ }^{i}\right) .
\end{aligned}
$$

Conversely, if the curvature tensor has the above form (6.7), it is easily seen that (6.3) is satisfied. Hence we have proved

THEOREM 6. A Sasakian manifold is of constant $\phi$-sectronal curvature if and only if the manifold satisfies the axiom of $\phi$-holomorphic 3-planes.

If a Sasakian manifold satisfies the axiom of $\phi$-holomorphic $(2 \gamma+1)$-planes, then it satisfies the axiom of $\phi$-holomorphic 3 -planes and hence it is of constant $\phi$-sectional curvature. Therefore we obtain

THEOREM 7. A Sasakıan manifold is of constant $\phi$-sectronal curvature if and only if the manifold satısfies the axiom of $\phi$-holomorphic $(2 r+1)$-planes.

In the proof of Theorem 7 , we have implicitly used the following Theorem 8.

THEOREM 8. Let $N$ be a $(2 r+1)$-dimensional totally geodesic submanifold of a Sasakian manifold $M^{2 n+1}$. If $N$ is $\phi$-holomorphic at one point $p$, then $N$ is invariant.

Proof. The van der Waerden-Bortolotti covariant derivative of $\eta_{2} \mathrm{C}_{x}{ }^{2}$ (see [5]), where $C_{x}{ }^{2}$ is a unit normal vector field. From (2.5) we have

$$
\nabla_{a}\left(\eta_{i} C_{x}{ }^{i}\right)=\phi_{\imath j} B_{a}{ }^{\imath} C_{x}{ }^{j}, \quad \nabla_{b} \nabla_{a}\left(\eta_{i} C_{x}^{i}\right)=-g_{b a} \eta_{i} C_{x}{ }^{2} .
$$

Because of the initial condition $\left(\eta_{i} C_{x}{ }^{i}\right)_{p}=\left(\phi_{i j} B_{a}{ }^{2} C_{x}{ }^{j}\right)_{p}=0$ the relation $\eta_{i} C_{x}{ }^{2}=$ $\phi_{i j} B_{a}{ }^{2} C_{x}{ }^{j}=0$ holds indentically on $N$. This equation shows that $N$ is invariant. 


\section{BIBLIOGRAPHY}

[1] D.E. BLAIR, Contact manifolds in Riemannian geometry, Lecture Notes in Mathematics Vol. 509 Springer Verlag, (1976).

[2] I. IshiHARA AND M. Kon, Contact totally umbilical submanifolds of a Sasakian space form, Ann. Mat. Pura Appl., CXIV (1977), 351-364.

[3] K. Ogive, On almost contact manifolds admitting axiom of planes or axiom of free mobility, Kōdai Math. Sem. Rep., 16 (1964), 223-232.

[4] S. SASAKI, Almost contact manifolds, Lecture Note I, Tôhoku University, (1965).

[5] K. YANO AND S. IshiHARA, Submanifolds with parallel mean curvature vector, J. Differential Geometry 6 (1971), 95-118.

[6] K. Yano AND M. Kon, Anti-1nvariant submanifolds of Sasakian space forms I, Tôhoku Math. Journ. 29 (1977), 9-23.

[7] K. YaNo AND M. Kon, Ant1-invariant submanifolds, Lecture Notes in Pure and Applied Mathematıcs Vol. 21, Marcel Dekker Inc., New York, (1976).

[8] K. YANO, M. KON AND I. IshIHARA, Ant1-1nvariant submanifolds with flat normal connection, to appear in J. Differential Geometry.

Tokyo Metropolitan Technical College

Higashi-OHI SHINAgAwA-KU

TOKYO, JAPAN 